\title{
Synthesis of Asymmetric One-Dimensional Pd on Au Bimetallic Nanostructures
}

Bishnu P. Khanal and Eugene R. Zubarev*

Department of Chemistry, Rice University, 6100 Main Street, Houston, TX 77005, United States

*Corresponding author: zubarev@ rice.edu

Supporting Information (SI) 

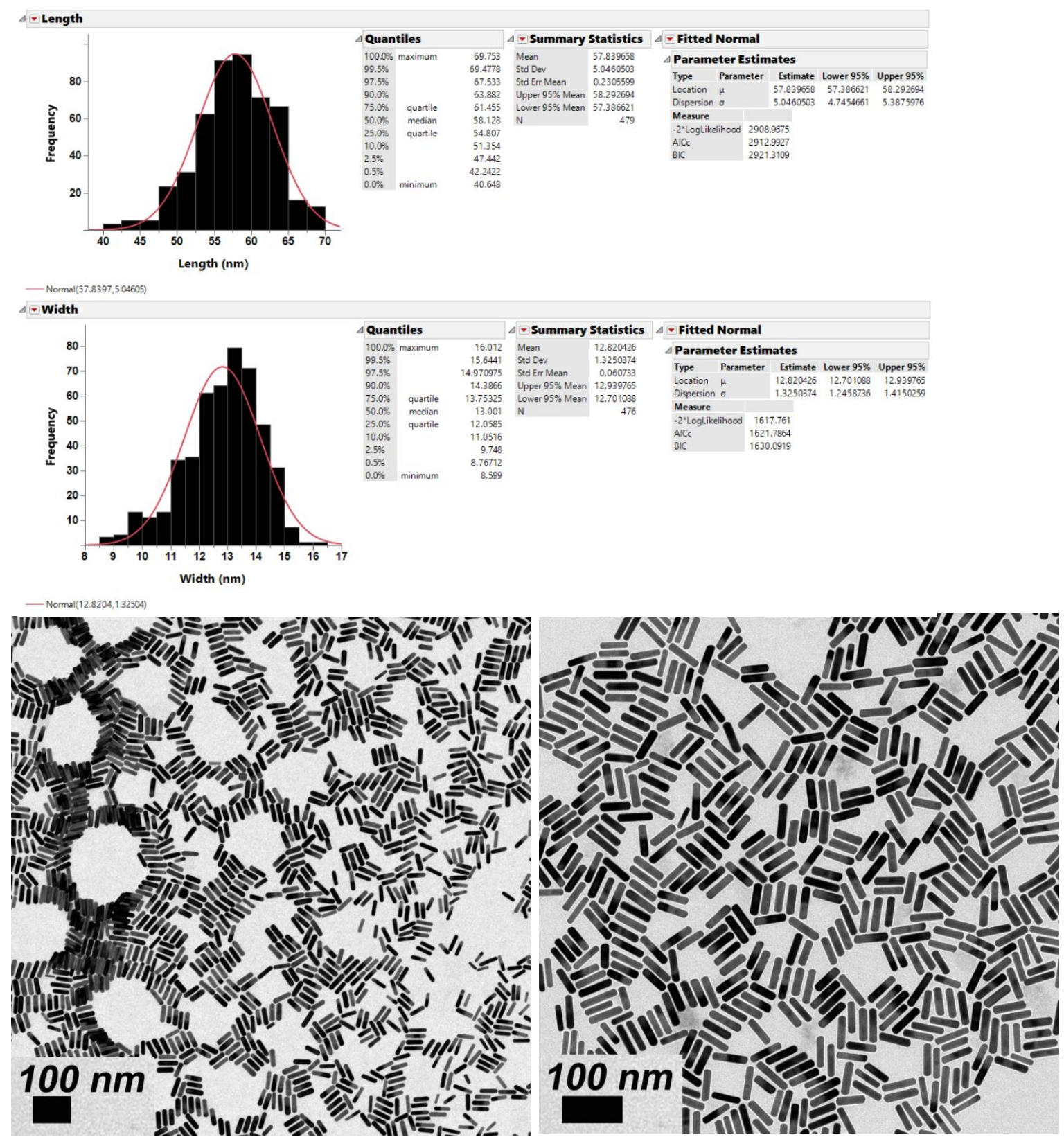

Figure SI 1. Histograms and detail statistical analysis for the size distribution of AuNRs. Representative TEM images of as synthesized AuNRs. TEM images of AuNRs from this figure and Fig 1 a. were used for the size distribution calculation. 

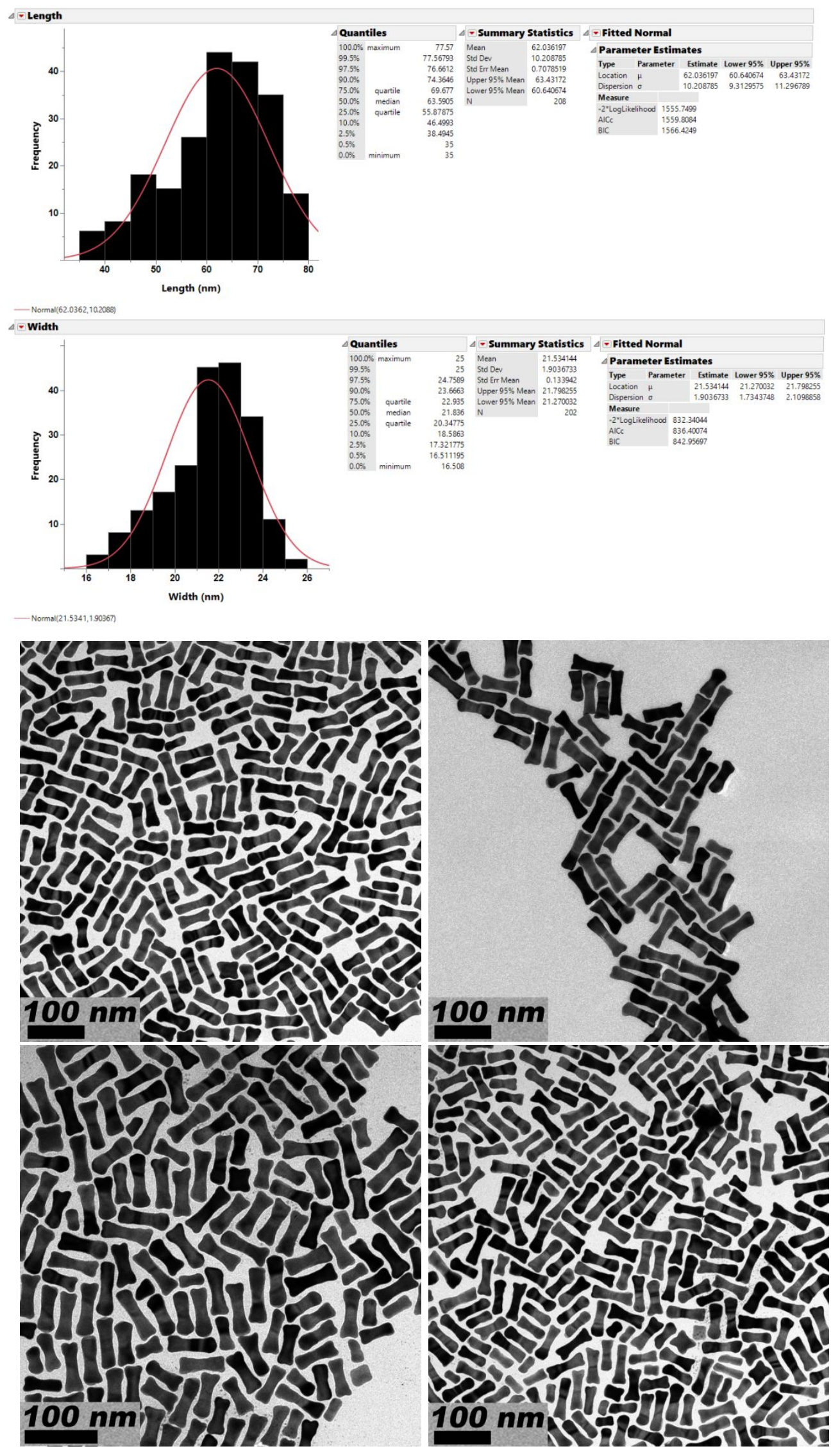

Figure SI 2. Histograms and detail statistical analysis for the size distribution of dogbone shaped AuNRs. Representative TEM images of dogbone shaped AuNRs used for the size 


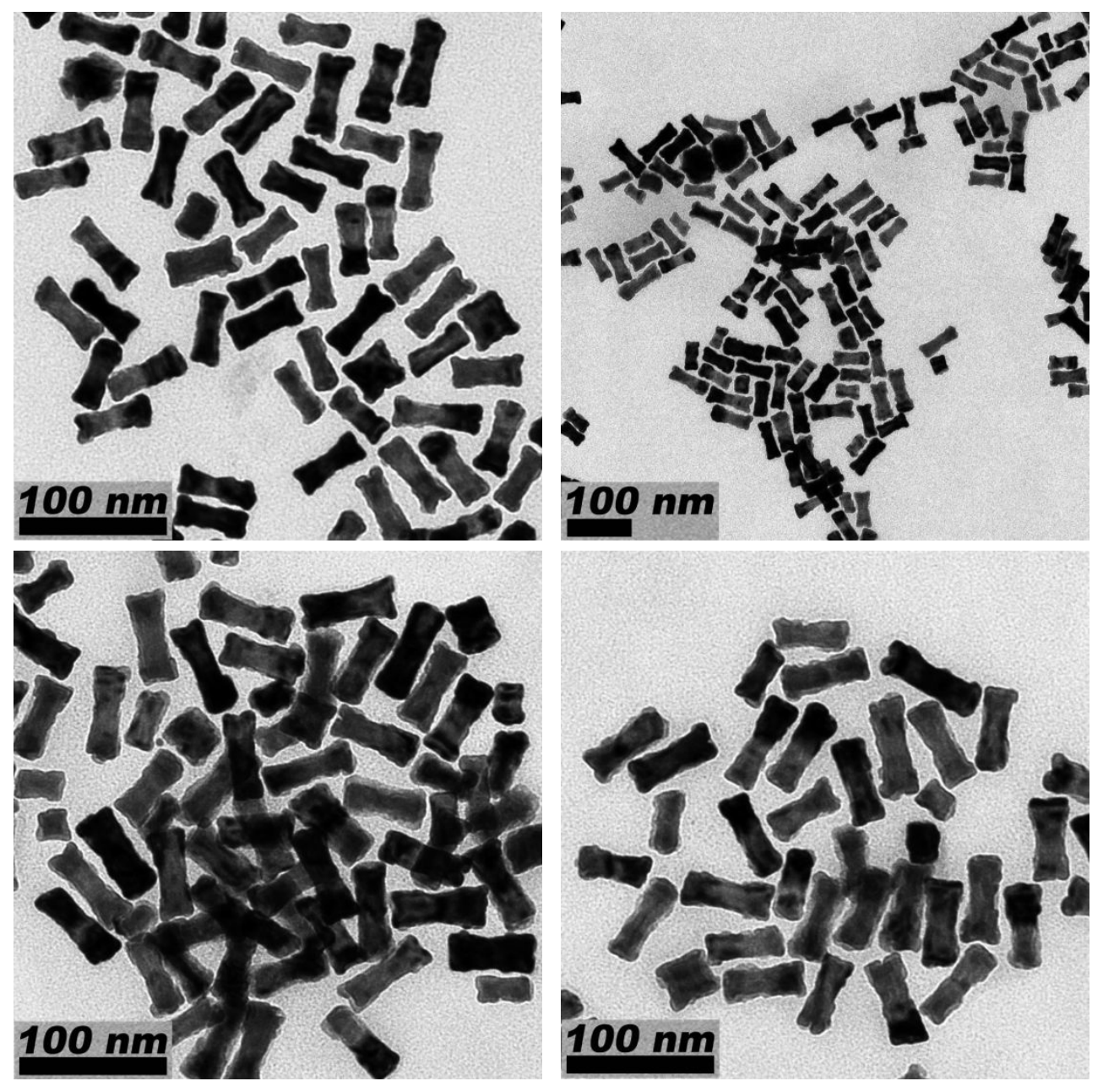

Figure SI 3. Additional TEM images showing the dog bone shape morphology of Pd@Au NRs during the initial stages of Pd depositon. 

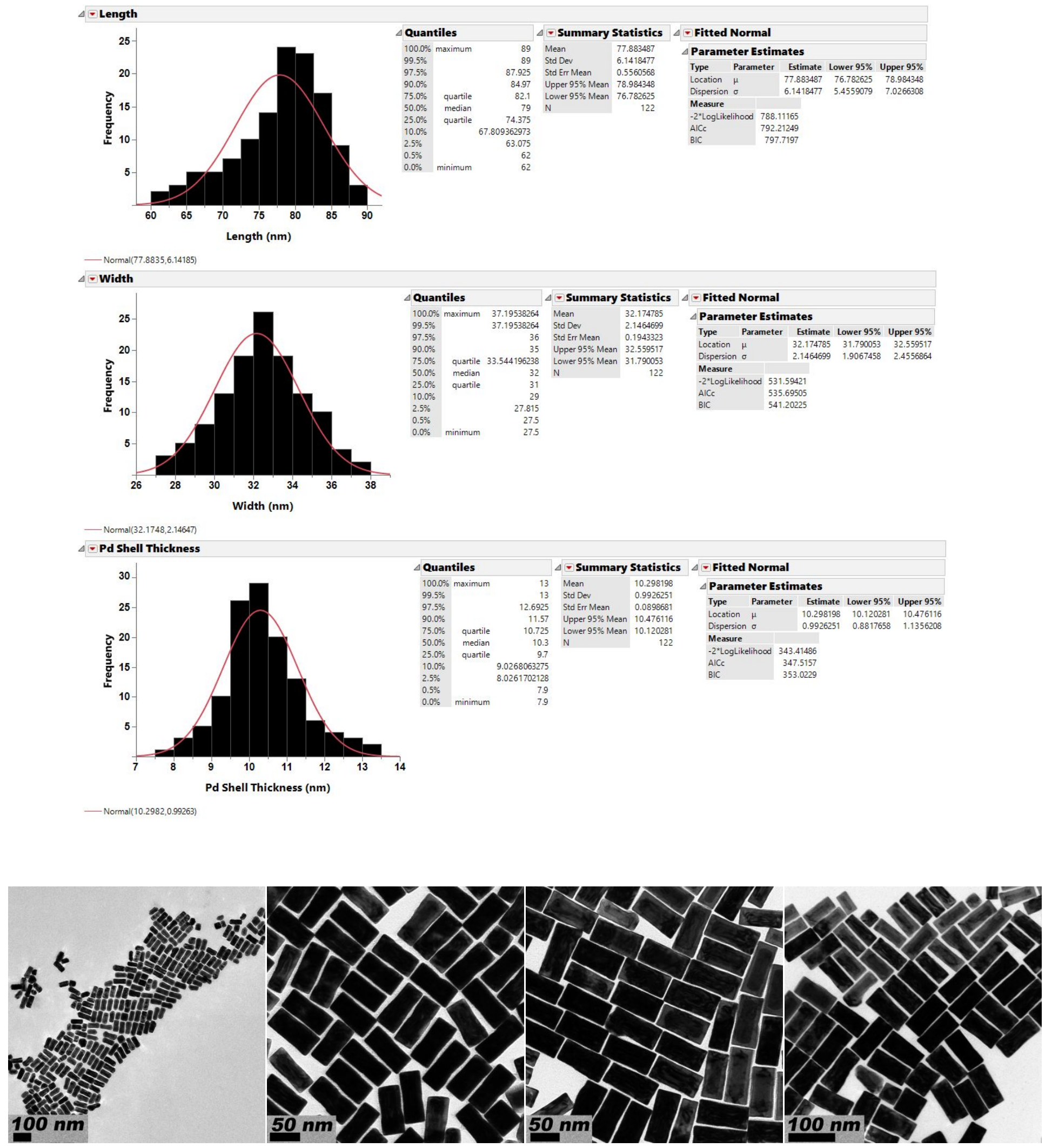

Figure SI 4. Detail statistical analysis for the size distribution of Au@Pd single crystalline nanorods and Pd shell thickness. Representative TEM images of Au@Pd NRs used for the size distribution calculation. 


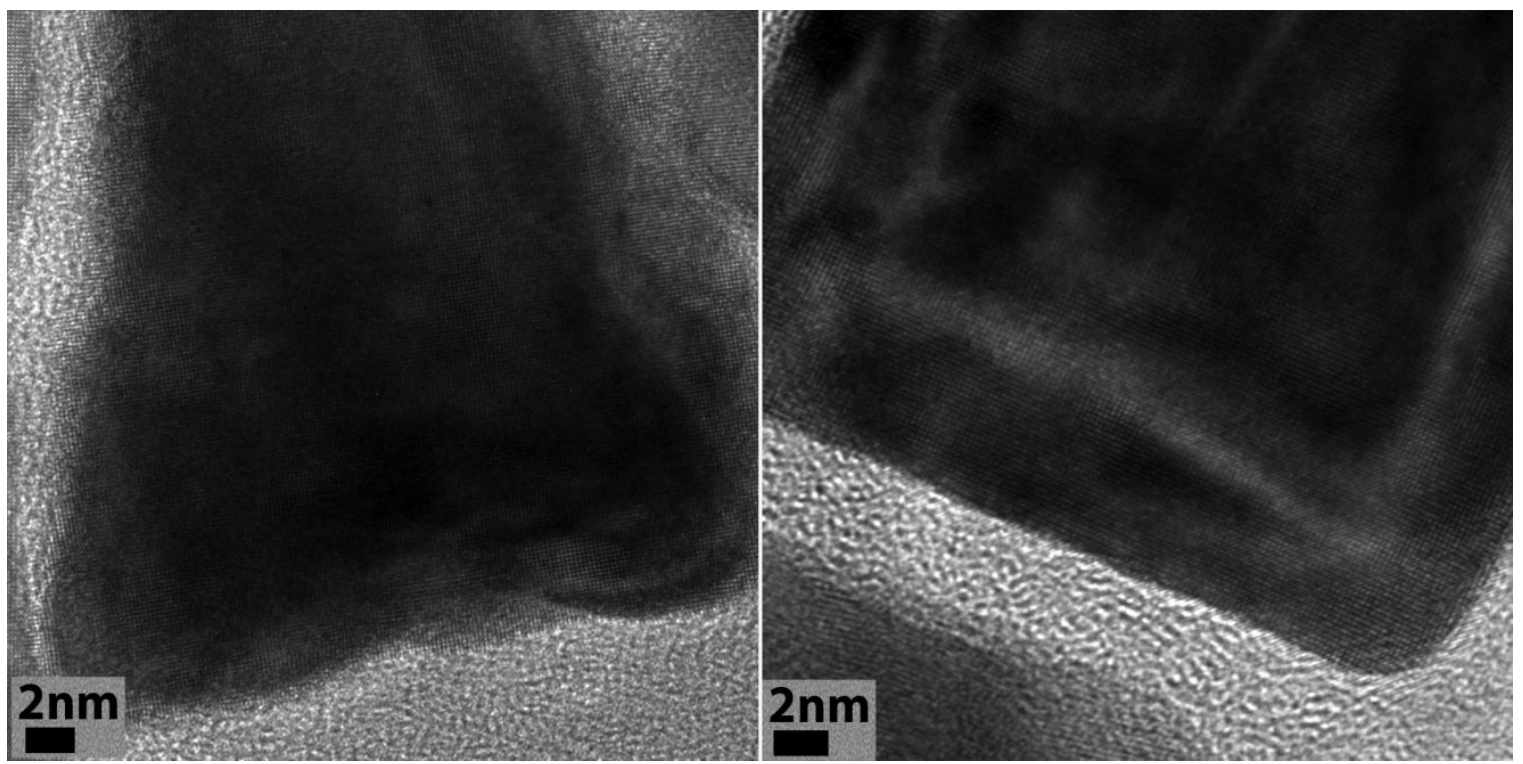

Figure SI 5. High resolution TEM TEM images Au@Pd NR showing the dog bone shape morphology (left) and complete transformation to rectangular shape (right). The Pd crystal

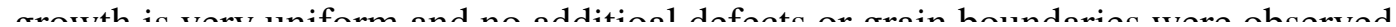




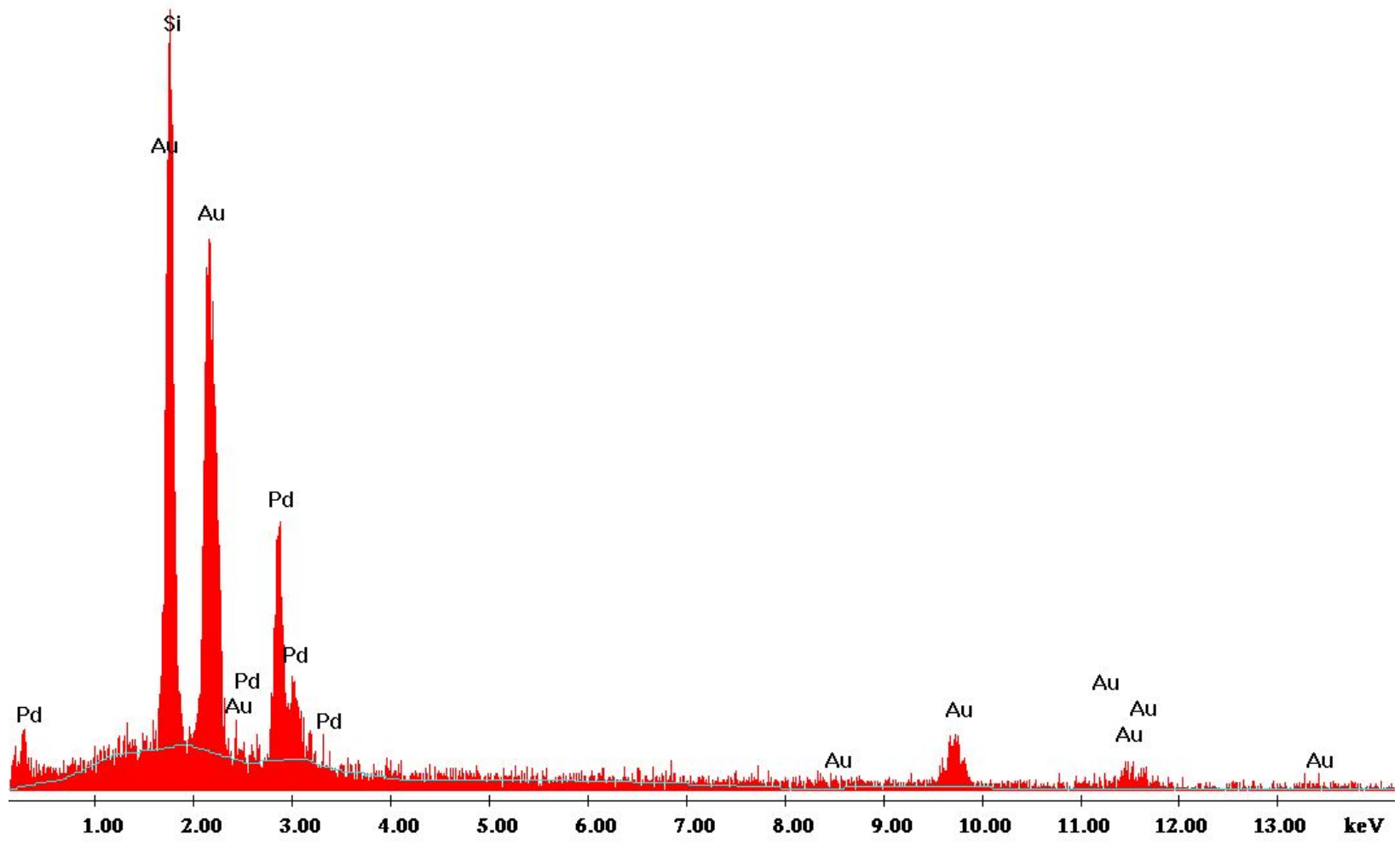

Figure SI 6. Energy dispersive X-ray spectroscopy (EDS) of Au@Pd NRs showing the respective noal from $\Delta_{11}$ and Dd matalc
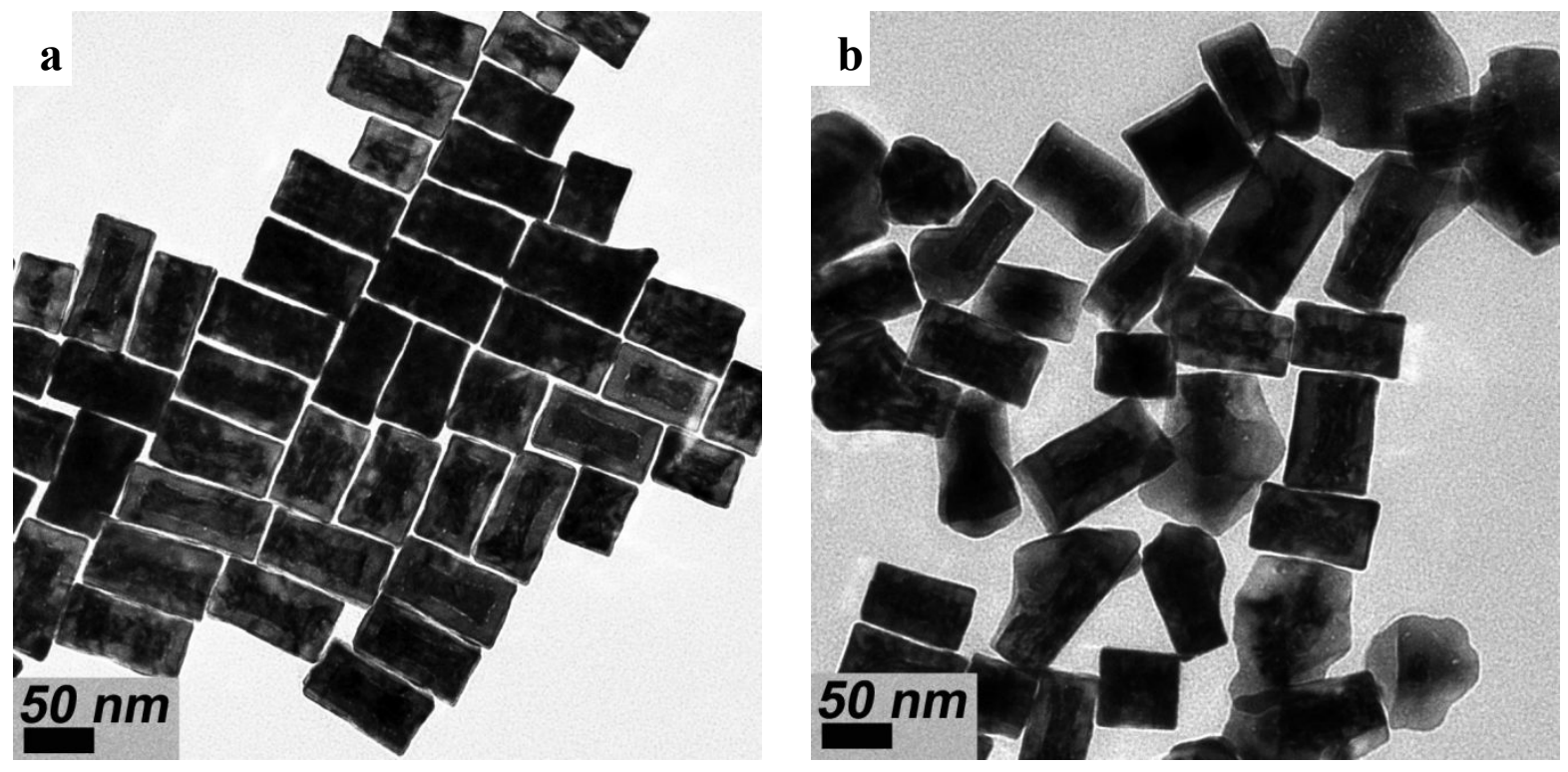

Figure SI 7. TEM image showing the $\sim 20 \mathrm{~nm}$ thick PdShell around AuNRs (a). Overgrowth of PdShell to irregular shape when attempted to grow thiker than $20 \mathrm{~nm}$ thick PdShell (b). 



Figure SI 8. TEM images showing irregular and non-uniform deposition of Pd on AuNR when

$\mathrm{Pd}(\mathrm{II})$ and AA concentraion was kept relatively higher ( $\sim 2$ times more concentration than normal) 

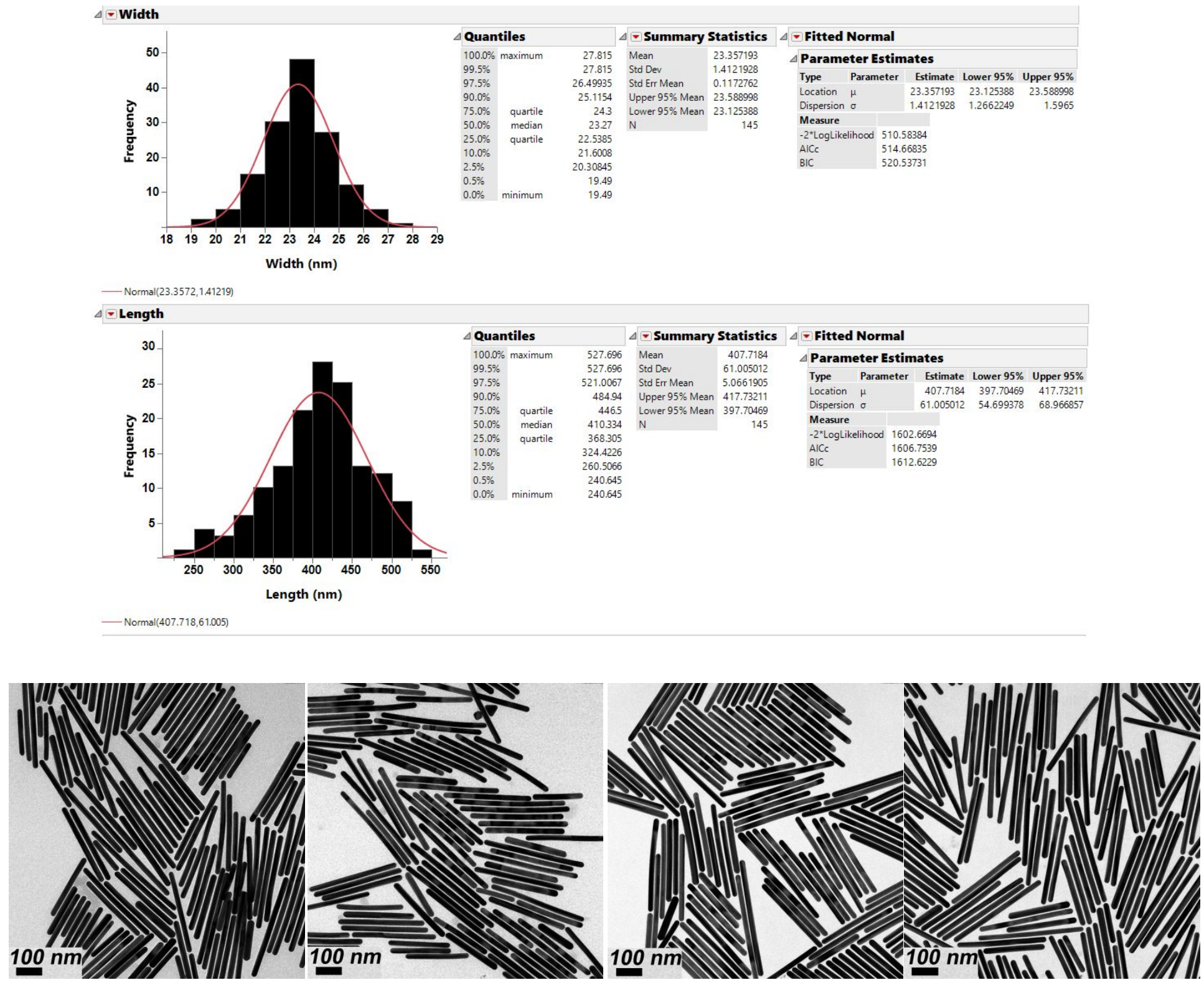

Figure SI 9. Detail statistical analysis for the size distribution of PHT AuNRs and representative TEM images of PHT AuNRs used for the size distribution calculation. 

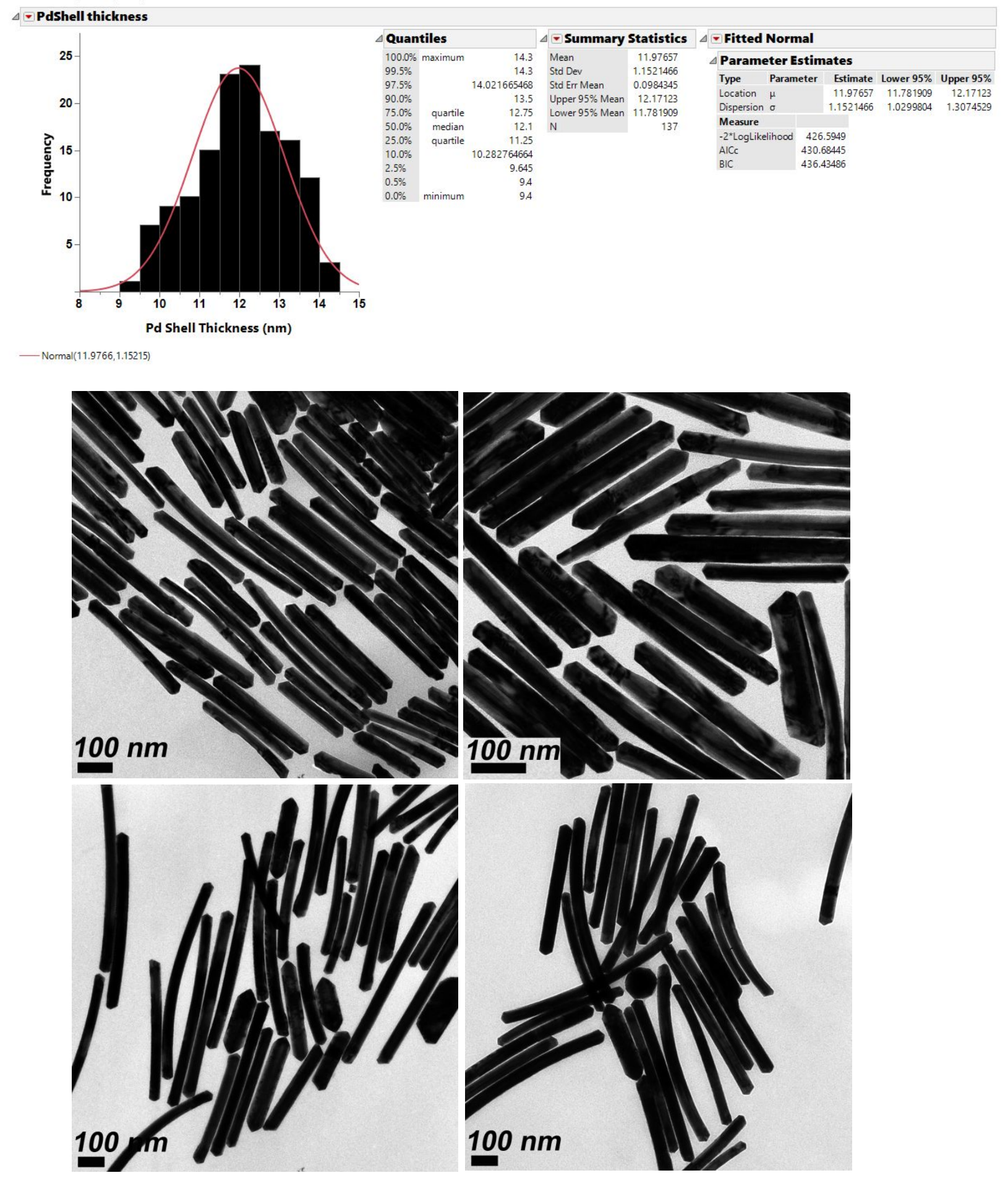

Figure SI 10. Statistical analysis for the Pd shell thickness around PHT AuNRs. Representative TEM images of Au@Pd PHT nanorods used for the size Pd shell thickness measurement. 


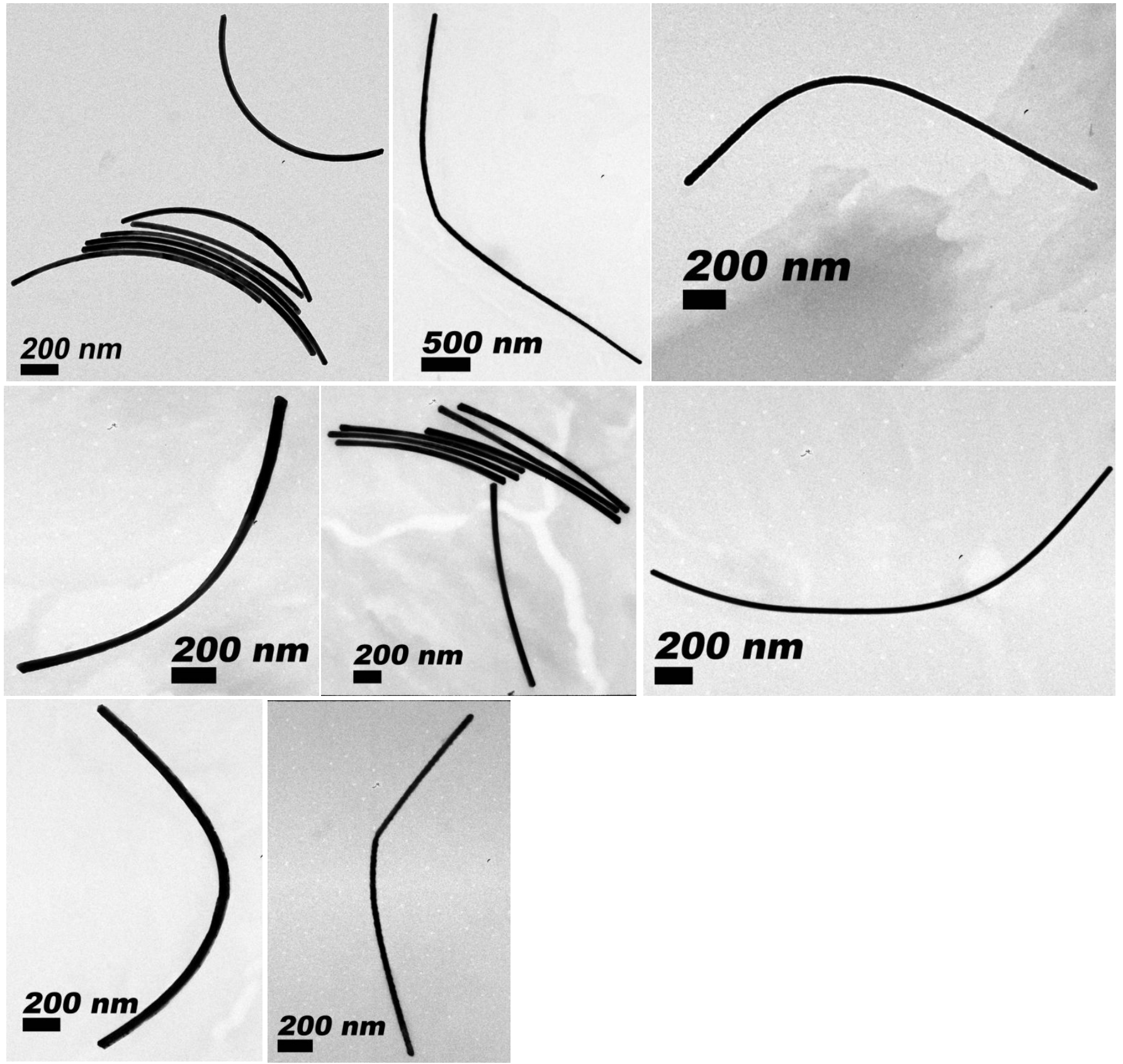

Figure SI 11. TEM images curved Pd@Au NRs of various lengths. The assemetric curved geometry was observed when Pd deposition was carried out on the AuNRs longer than $\sim 800 \mathrm{~nm}$. 
